\title{
Corneal hypoaesthesia in asymptomatic familial distichiasis
}

\author{
I KREMER, D WEINBERGER, S COHEN, AND I BEN SIRA \\ From the Department of Ophthalmology, Beilinson Medical Center, Petah Tiqva, Tel Aviv University Sackler \\ School of Medicine, Israel
}

SUMmARY A 3-year-old child, his mother, and his uncle were found to have asymptomatic distichiasis accompanied by corneal hypoaesthesia. Slit-lamp examination of the mother and her brother revealed normal corneas, but bilateral punctate epithelial staining was found in the child. In addition the child suffered from corneal erosion in his left eye due to decreased tear secretion after left occipital craniotomy and $\mathrm{C} 1$ laminectomy.

Distichiasis is an extremely rare congenital anomaly in which an accessory row of eyelashes occurs along the posterior border of the lid margin in the position of the meibomian glands. ${ }^{1-5}$ A strong hereditary factor has been noted in many reports, ${ }^{5-8}$ and the condition is apparently a dominant hereditary characteristic, since transmission is direct without sex predilection. ${ }^{5}$ Sometimes distichiasis is accompanied by other congenital anomalies, and in rare cases congenital ectropion, entropion, ptosis, and maxillofacial dysostosis occur simultaneously. ${ }^{s-7}$ Ocular irritation is not so common as one would think, ${ }^{1-13} \mathrm{a}$ fact which is probably due to the shortness and thinness (lanugo-like) of these accessory lashes. However, distichiasis may sometimes cause great discomfort and require surgical treatment. ${ }^{14} \mathrm{We}$ describe three family members with distichiasis who were completely asymptomatic; corneal hypoaesthesia was found in each of them. This hypoaesthesia, in addition to the quality of the aberrant eyelashes, most probably contributed to the absence of any irritation. We wonder whether this hypoaesthesia is secondary to the adaptation of the corneal sensory nerve endings, to the chronic mild epithelial trauma caused by the aberrant eyelashes, or to a congenital anomaly accompanying the distichiasis.

\section{Case report}

A 3-year-old boy was referred to our department

Correspondence to $\mathrm{D}$ Weinberger, MD, Department of Ophthalmology, Beilinson Medical Center, Petah Tiqva 49100, Israel. owing to blurring of vision of his left eye three weeks after left suboccipital craniotomy and C1 laminectomy had been performed for a cerebellar mixed glioma occupying the whole posterior fossa. Examination of each eye revealed distichiasis of the upper and lower lids of both sides. The accessory cilia on the posterior margin were much thinner and lighter in colour than those on the anterior margin of the lid borders. Some were so light and thin as to be seen only with the slit-lamp. There were 10 aberrant cilia in the right upper lid and 12 in the right lower lid; 12 aberrant cilia were found in the left upper lid and 15 in the left lower lid. All the cilia pointed backwards and impinged on the eyeball. Both corneas showed punctate epithelial staining in their inferior half. In addition a large epithelial erosion was found in the paracentral area of the left cornea. No other abnormal findings were seen in the anterior and posterior segments. Corneal sensitivity testing by the corneal aesthesiometer disclosed very low values (1.5 units in both eyes). Schirmer test values were found to be above $15 \mathrm{~mm}$ in the right eye and below $10 \mathrm{~mm}$ in the left. The corneal erosion was treated by lateral tarsorrhaphy combined with local treatment with chloramphenicol ointment (5\%) four times daily.

After two weeks the corneal erosion healed completely but epithelial staining still remained in both eyes. Therefore electroepilation of the accessory eyelashes was performed, after which procedure the punctate staining disappeared almost completely. As the Schirmer test results were found to be improved (above $10 \mathrm{~mm}$ ) in the left eye, the lateral tarsorrhapy was opened and the local treatment stopped. On follow-up examinations the aberrant eyelashes 


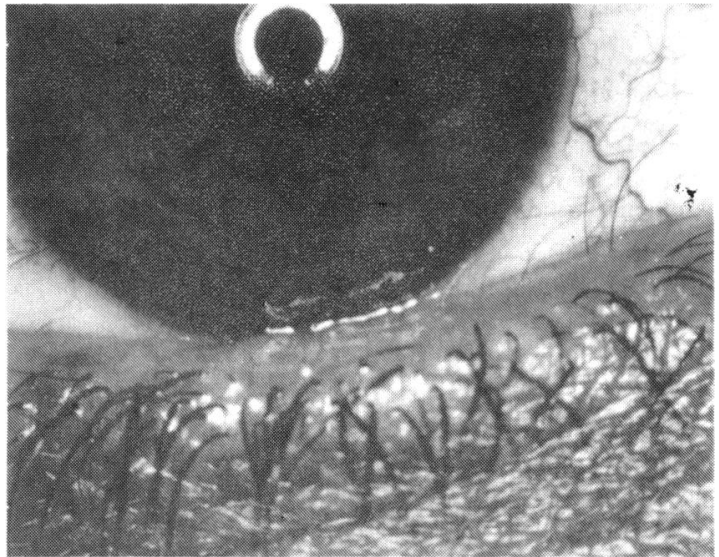

Fig. 1 Distichiasis in upper lid of the mother's right eye.

reappeared and the child was completely asymptomatic, but very mild punctate staining was still noticed in the lower part of both corneas. It was decided to keep the child on artificial tears treatment six times daily in both eyes.

We checked the child's family members and found that his mother and her brother, 30 years and 23 years old respectively, also had distichiasis in both eyelid margins, but without corneal lesions (Figs 1,2). The mother's brother had no children and the child had no siblings. no other family members were found to have distichiasis, and their general physical and ophthalmological examination revealed no abnormal findings.

\section{Discussion}

In distichiasis the aberrant lashes are usually small and very soft and fine, showing little pigmentation, but they may at times be as fully developed as the outer row. ${ }^{12}$ Anatomical investigation has verified that they replace the meibomian glands, which become modified and take on hair-bearing functions. ${ }^{8-11}$ Sometimes these glands appear to lose all their usual characteristics, being entirely replaced by the follicles of cilia into which sebaceous glands open, but at other times some typical meibomian acini remain while opening into the follicles of the anomalous cilia like normal sebaceous glands. ${ }^{10-13}$ Begle $^{10}$ suggested that this condition might be atavistic, as it is common in animals. Hereditary cases have been reported by several authors, mainly in the older literature..$^{5-7}$ As has been mentioned, the condition is usually transmitted as a dominant characteristic. ${ }^{5}$ Pico $^{5}$ has described a family with 10 individuals who had distichiasis, eight of whom had associated ectropion and the other two distichiasis as an isolated anomaly. ${ }^{5}$ Other congenital anomalies

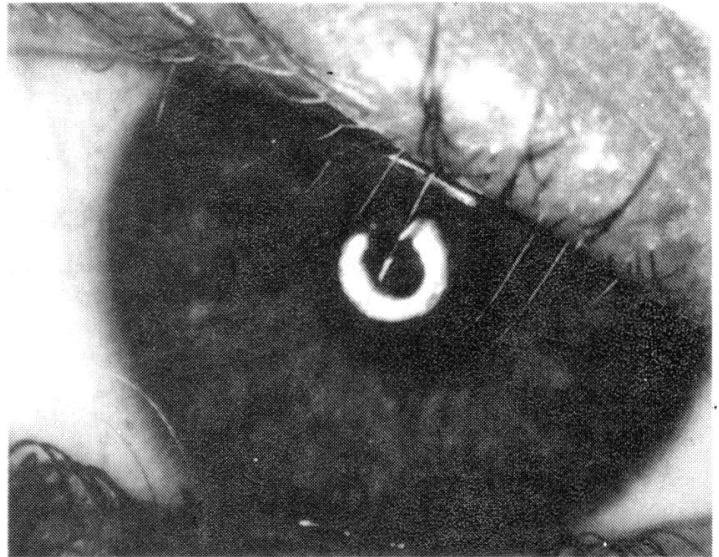

Fig. 2 Distichiasis in upper lid of mother's left eye.

have been described as being found together with distichiasis. ${ }^{467}$

The clinical importance of distichiasis lies in the irritation caused to the cornea, ${ }^{1-35}$ but as was found in the family reported here and in some others mentioned in the literature the irritation is usually less than might be expected; often this anomaly is completely asymptomatic owing to the fineness of the eyelashes $^{1-3}$ and probably, we assume, to corneal hypoaesthesia or to adaptation by the corneal nerve endings - a hypothesis difficult to prove. Fox ${ }^{2}$ described a case of distichiasis accompanied by slight corneal hypoaesthesia, but others have not mentioned this interesting fact in the relatively few reports written on this subject in recent years. ${ }^{3-7}$ If the irritation is mild and the abnormal eyelashes are few, electroepilation may solve the problem, but, if a complete row is present and the irritation is severe enough, a plastic operation for trichiasis is indicated. ${ }^{14}$ In the family described here two brothers and their sister were asymptomatic, the child of the last having distichiasis with corneal complications due to deficiency of tearing reflex. It is interesting to note that all of them were found to have corneal hypoaesthesia when tested with the corneal aesthesiometer.

\section{References}

1 Scheie HG, Albert DM. Distichiasis and trichiasis: origin and management. Am J Ophthalmol 1966; 61: 718-20.

2 Fox SA. Distichiasis. Am J Ophthalmol 1962; 53: 14-8.

3 Dohetry WB. Congenital distichiasis. Am J Ophthalmol 1927; 10: 248-50.

4 Bader A. Aplasia congenitalis glandularum meibomii palpebrae. Graefes Arch Klin Exp Ophthalmol 1950; 150: 411-3.

5 Pico G. Congenital ectropion and distichiasis: etiologic and hereditary factors. A report of cases and review of literature. $\mathrm{Am}$ J Ophthalmol 1959; 47: 363-87.

6 De Voe AG, Horwich H. Congenital entropion and tetra- 
stichiasis of the upper lids, palpabral hyperpigmentation and mental deficiency. Arch Ophthalmol 1954; 52: 865-70.

7 Halberg GP, Paunessa JM. An incomplete form of mandibulofacial dysostosis (Franceschetti's syndrome). Br J Ophthalmol 1949; 33: 709-13.

8 Frolowa V. Sur le distichiasis congenita vera. Ann Occulist (Paris) 1927; 164: 433-42.

9 Halvertsma KTA. Distichiasis congenita vrai. Arch Ophtalmol (Paris) 1935; 52: 738-48.

10 Begle HL. Klinisch-anatomischer Beitrag zue Kenntnis der Distichiasis congenita. Arch Augenheilkd 1913; 74: 62-78.
11 Stephenson S. A case of congenital distichiasis. Trans Ophthalmol Soc UK 1902; 22: 192-3.

12 Elschnig A. Distichiasis: case presentation. Wien Klin Wochenschr 1912; 25: 1144.

13 Jeanselme E, Morax V. Distichiasis familial et héréditaire. Ann Oculist (Paris) 1904; 132: 66.

14 Dortzbach RK, Buterna RT. Excision of dystichiasis eyelashes through tarsoconjunctival trapdoor. Arch Ophthalmol 1978, 96: 111-2.

Accepted for publication 17 June 1985. 